\title{
The (methylation) reader
}

$N^{6}$-methyladenosine $\left(\mathrm{m}^{6} \mathrm{~A}\right)$ is a prevalent internal modification of eukaryotic mRNAs, which can be reversed and could be involved in the regulation of mRNA metabolism, translation and turnover. Now, Wang et al. report that YTH domain family 2 (YTHDF2) selectively recognizes $\mathrm{m}^{6} \mathrm{~A}$ and functions as a 'reader' protein that promotes mRNA degradation.

The YTH domain family is widespread in eukaryotes and binds to single-stranded RNA. The authors focused on YTHDF2 given its high selectivity for $\mathrm{m}^{6} \mathrm{~A}$ - it showed a 16-fold higher binding affinity to methylated RNA compared to unmethylated RNA. By analysing YTHDF2-RNA complexes, they found that most of the RNA was mRNA. Moreover, YTHDF2 was found to preferentially bind to the stop codon region, the $3^{\prime}$ untranslated region and the coding region, which suggests that YTHDF2 might have a role in mRNA stability and/or translation.

The authors then used 콜. ribosome profiling and 흘 RNA sequencing to 윳 assess how YTHDF2

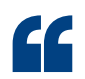

the first functional characterization of an $\mathrm{m}^{6} \mathrm{~A}$ reader protein affects the loading of its target $\left(\mathrm{m}^{6} \mathrm{~A}\right.$ containing) mRNAs onto ribosomes and their cytoplasmic levels. After knockdown of YTHDF2 using siRNA, cells accumulated increased amounts of methylated YTHDF2 mRNA targets, but there was no obvious difference in their association with ribosomes, which indicates that YTHDF2 has a primary role in mRNA degradation. Indeed, YTHDF2 knockdown led to prolonged lifetimes of its target mRNAs. mRNAs exist in three pools: nonribosome mRNA-protein particles (mRNPs), a translatable mRNA pool associated with ribosomal subunits and actively translating polysomes. When YTHDF2 was knocked down, the ratio of methylated-to-unmethylated mRNAs was decreased in mRNPs and increased in the translatable pool, which suggests that YTHDF2 promotes the distribution of $\mathrm{m}^{6} \mathrm{~A}$-containing mRNAs to mRNPs. This YTHDF2-dependent localization to non-translatable pools was confirmed by analysing the distribution of two specific mRNAs. YTHDF2 consists of a carboxy-terminal
RNA-binding domain (C-YTHDF2) and a Pro-Gln-Asn-rich aminoterminal domain (N-YTHDF2), which were both required for YTHDF2-mediated mRNA decay. When analysing the subcellular localization of YTHDF2, the authors observed that full-length YTHDF2 and N-YTHDF2 both localized to processing bodies (P bodies), but C-YTHDF2 alone did not. Furthermore, one of its target mRNAs colocalized with full-length YTHDF2 and C-YTHDF2, but not with N-YTHDF2. This indicates that the $\mathrm{N}$-terminal domain of YTHDF2 is required for localization of the protein, in complex with mRNAs, to $\mathrm{P}$ bodies.

Together, these results led the authors to suggest a model in which C-YTHDF2 selectively recognizes $\mathrm{m}^{6} \mathrm{~A}$-containing mRNAs that are not being actively translated, and then $\mathrm{N}-$ YTHDF2 mediates the relocation of YTHDF2- $\mathrm{m}^{6} \mathrm{~A}-\mathrm{mRNA}$ complexes to specialized mRNA degradation machineries such as $\mathrm{P}$ bodies.

This work provides the first functional characterization of an $\mathrm{m}^{6} \mathrm{~A}$ reader protein and a main biological function of the $\mathrm{m}^{6} \mathrm{~A}$ modification on mRNA, which is to alter the distribution and stability of mRNAs.

\section{Kim Baumann}

ORIGINAL RESEARCH PAPER Wang, X. et al. $\mathrm{N}^{6}$-methyladenosine-dependent regulation of messenger RNA stability. Nature http://dx.doi. org/10.1038/nature12730 (2013) 